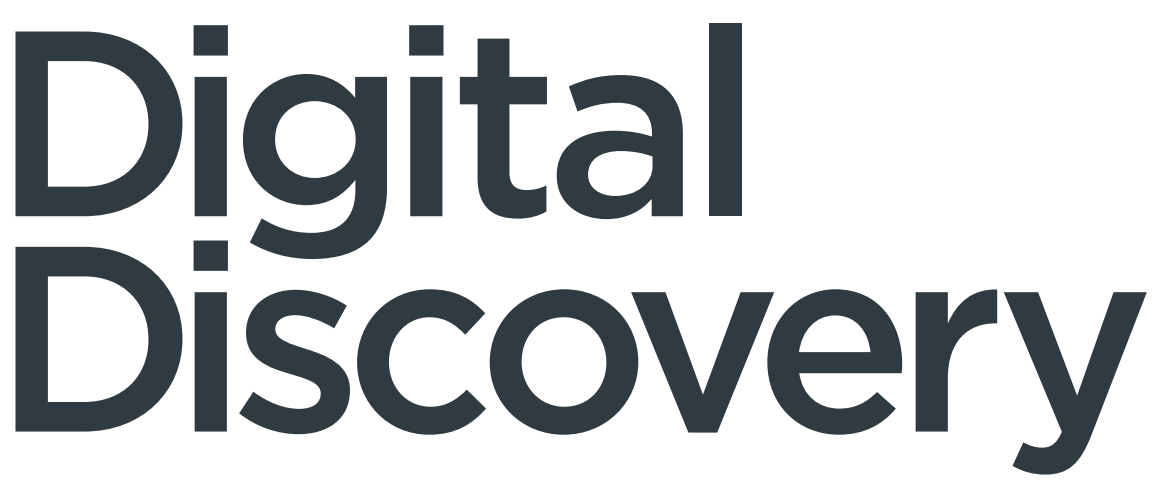

Number 2

April 2022

Pages 73-174

rsc.li/digitaldiscovery

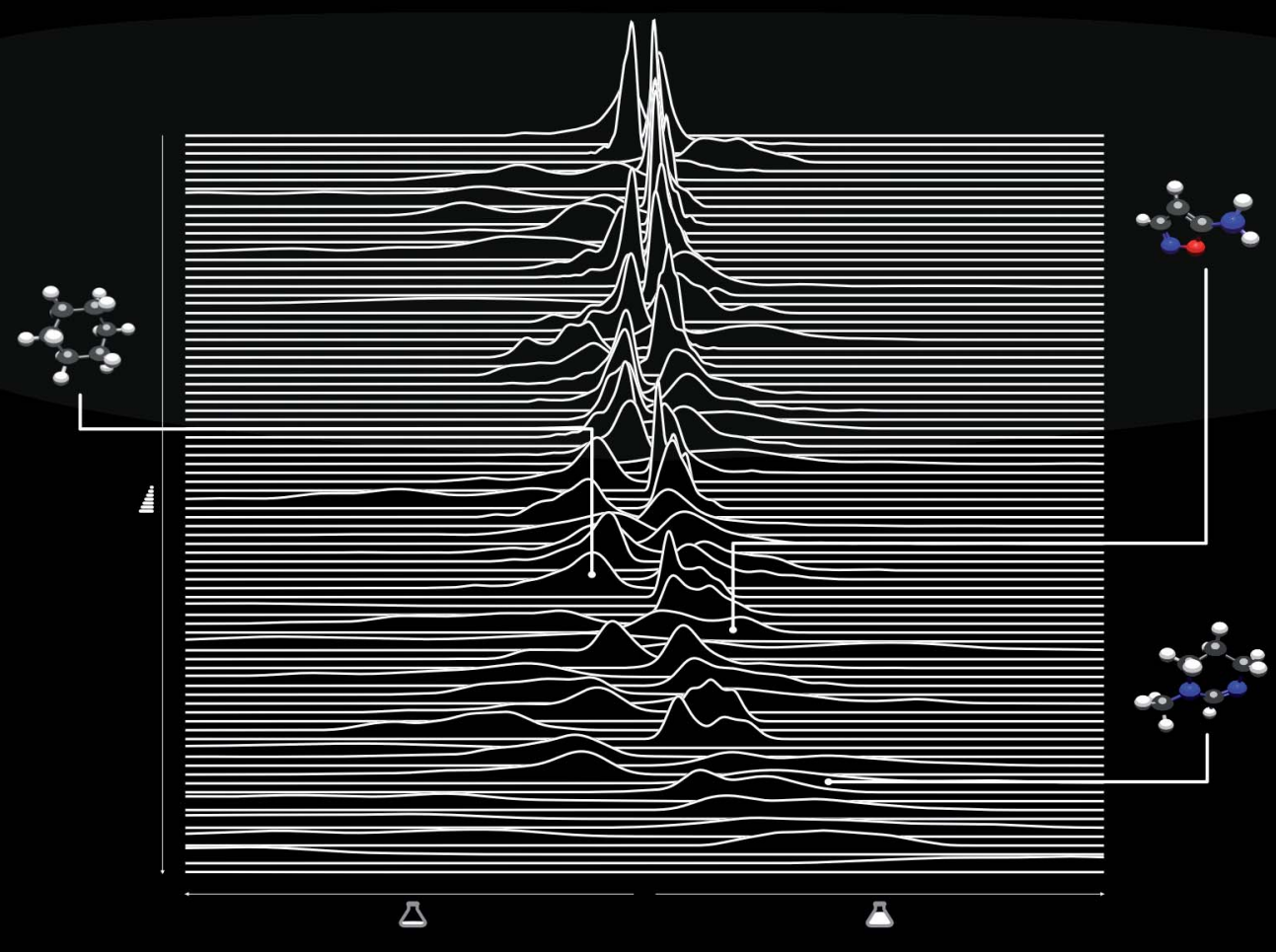


Check for updates

Cite this: Digital Discovery, 2022, 1, 91

\title{
Reaction classification and yield prediction using the differential reaction fingerprint DRFP
}

\author{
Daniel Probst, (D) *a Philippe Schwaller (iD ${ }^{b}$ and Jean-Louis Reymond (D) *a
}

Predicting the nature and outcome of reactions using computational methods is a crucial tool to accelerate chemical research. The recent application of deep learning-based learned fingerprints to reaction classification and reaction yield prediction has shown an impressive increase in performance compared to previous methods such as DFT- and structure-based fingerprints. However, learned fingerprints require large training data sets, are inherently biased, and are based on complex deep learning architectures. Here we present the differential reaction fingerprint DRFP. The DRFP algorithm takes a reaction SMILES as an input and creates a binary fingerprint based on the symmetric difference of two sets containing the circular molecular $n$-grams generated from the molecules listed left and right from the reaction arrow, respectively, without the need for distinguishing between reactants and reagents. We show that DRFP performs better than DFT-based fingerprints in reaction yield prediction and other structure-based fingerprints in reaction classification, reaching the performance of state-of-the-art learned fingerprints in both tasks while being data-independent.

Received 26th August 2021
Accepted 12th January 2022

DOI: $10.1039 / \mathrm{d} 1 \mathrm{dd} 00006 \mathrm{c}$

rsc.li/digitaldiscovery
Recently, these limitations were overcome by applying a natural language processing-inspired transformer architecture as a means to learn the vector-embedding of reactions. ${ }^{\mathbf{1 1}}$ However, this approach comes with several drawbacks as well: training such a learned fingerprint requires large amounts of data of acceptable quality, the trained model must be retrained when new data becomes available, and the training and evaluation of the model requires specialised hard- and software to become computationally tractable-posing a challenge to accessibility and reproducibility. Due to the nature of artificial neural networks (ANNs), learned fingerprints are also challenging to interpret. In the case of the transformer-based model introduced by Schwaller et al., a careful analysis of attention weights is required. ${ }^{11}$

An important outcome of a chemical reaction is its yield, the percentage of successfully converted reactants into the desired product. Computational methods for predicting such yields are highly valuable in synthesis-planning, where high yields are of paramount importance-especially in multi-step reactions. Earlier work used physics-based descriptors or structure-based molecular fingerprints to classify chemical reactions or predict reaction yields. ${ }^{6,17,19}$ While physics-based descriptors require compute-intensive calculations that involve the approximation of the N-body wave function of molecules, structure-based descriptors that are calculated from the molecular graph fail to generalize between data sets. ${ }^{11}$ Similar to the problem of reaction classification, the recent availability of large data sets and the resurgence of ANNs, deep learning-based learned fingerprints have been introduced as an alternative to earlier methods, outperforming them by considerable
${ }^{a}$ Department of Chemistry and Biochemistry, University of Bern, Freiestrasse 3, 3012 Bern, Switzerland.E-mail: daniel.probst@dcb.unibe.ch; jean-louis.reymond@unibe. ch

${ }^{b} I B M$ Research - Europe, Säumerstrasse 4, 8803 Rüschlikon, Switzerland 
margins. ${ }^{10}$ However, these methods also suffer the same drawbacks as their counterparts used for reaction classification.

Here we report a molecular fingerprint for chemical reactions called differential reaction fingerprint $(D R F P)$, which is computed from circular substructures in the reaction SMILES without the need for a training data set. Compared to the approach introduced by Schneider et al. ${ }^{17}$ DRFP does not apply weights based on atom-mapping to differentiate between reactants and reagents, does not require the calculation of molecular properties for the reagents, and does not apply arithmetic operations on individual molecular fingerprints, such as the atom pair fingerprint. We show that DRFP performs as well as learned fingerprints for the tasks of reaction classification and yield prediction.

\section{Results and discussion}

\subsection{Fingerprint design}

Here we present the differential reaction fingerprint $(D R F P)$ for reaction search and categorization as well as yield prediction. The reaction fingerprint $D R F P$ borrows the creation of circular substructures from a molecule and the subsequent hashing of their SMILES representations from the chemical fingerprints ECFP and MHFP, respectively (see Fig. 1 and molecular $n$ grams), where circular substructures for a molecule are created by extracting the neighbourhoods of a given radius $r$ from the molecular graph for each atom in the molecule. ${ }^{20,21}$ However, as reaction SMILES consist of multiple molecules in the form REACTANTS $>$ REAGENTS > PRODUCTS (Fig. 1a), three additional steps have to be introduced: (I) the reagents are added to the reactants, resulting in the representation REACTANTS + REAGENTS $\gg$ PRODUCTS (Fig. 1b); (II) circular substructures are extracted from each molecule (Fig. 1c), resulting in two sets of molecular $n$-grams $R$ and $P$ (Fig. $1 \mathrm{~d}$, red and blue circles); (III) the symmetric difference of the two sets $S=R \Delta P$ is taken (Fig. 1d, shaded areas of circles), hashed using an arbitrary hash function with a sufficiently low collision probability (Fig. 1e), and then folded into a fix-length binary vector using the hash function $h(k)=k \bmod d$, where $k \in S$, and $d$ is the desired dimensionality of the fingerprint (Fig. 1f). Hashing and folding are required to transform the set of SMILES, which can differ in cardinality given different input reactions, into a binary vector of predefined dimensionality $d$ that is independent from the input reaction. Binary vectors require little space in memory and can be processed by most machine learning methods.

Similar to the transformer-based learned fingerprint, DRFP does not distinguish between reactants and reagents, and accepts an arbitrary number of molecules on both sides of the chemical equation. Given this conceptually simple fingerprint, we show that its performance, when applied to tasks mentioned in the introduction, rivals or even surpasses that of state-of-theart methods while using minimal non-specialised computational resources and no specialised hard- or software (see Computational resources). The fingerprint requires an unannotated, non-atom-mapped reaction SMILES as input and embeds this molecular representation from reaction SMILES space into an arbitrary low dimensional binary metric space

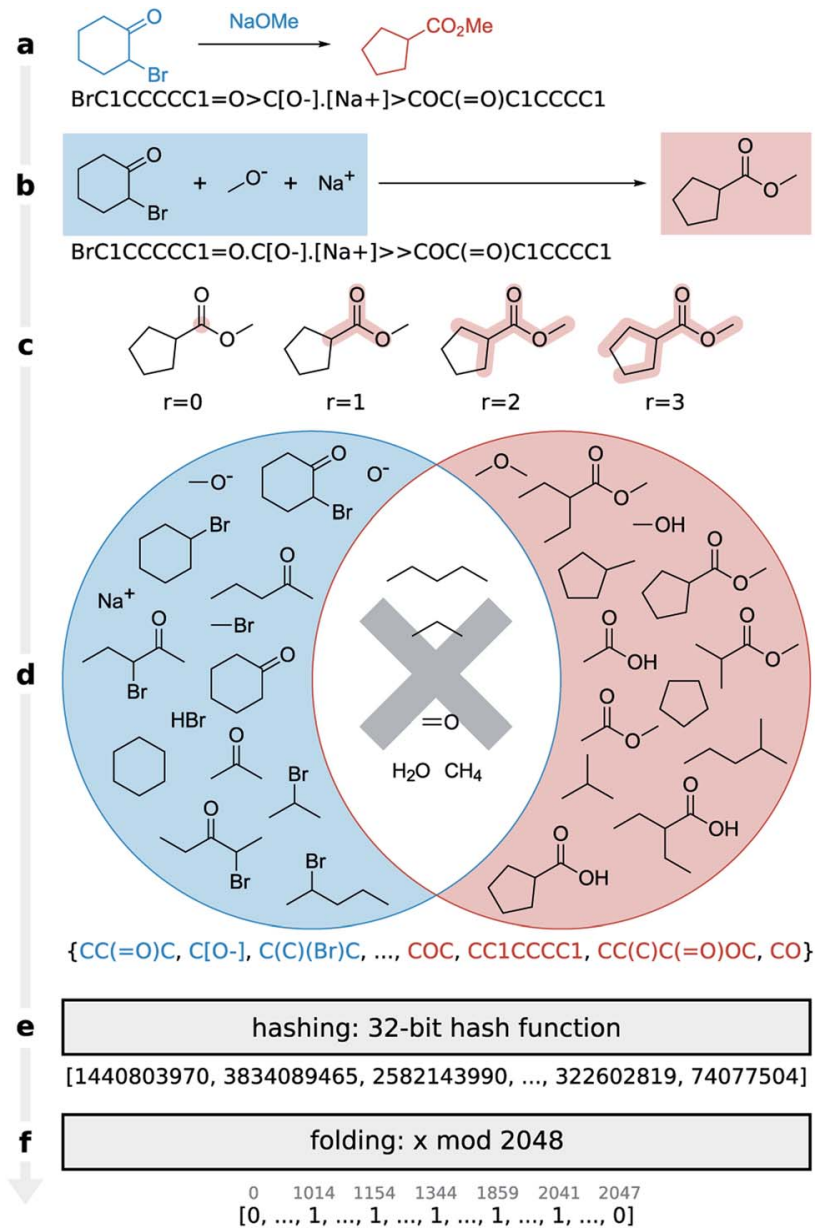

Fig. 1 Encoding a Favorskii rearrangement as a DRFP fingerprint. (a) The input reaction where the reactant is written to the left, the reagent above, and the product to the right of the arrow. The respective SMILES representation, which is used as the input is written below. (b) DRFP does not separate reactants and reagents, a distinction which is often ambiguous. This is reflected in the reaction drawing and the associated SMILES, where the reagents have been moved to the reactants. (c) The algorithm extracts all circular substructures with radii of $0,1,2$, and 3 , as well as all rings from the reactants and products, and stores them as two SMILES-encoded sets of molecular $n$-grams (d). Next, the symmetric difference of the two sets is calculated and stored as the final set. (e) This final set of molecular $n$-grams is then hashed to a vector of 32-bit integers and (f) folded into a fixed-length binary vector using a modulo operation.

through set operations and subsequent hashing and folding. We show that a $k$-NN classifier trained with DRFP outperforms those trained on existing, non-learned fingerprints and rivals or surpasses the performance of learned fingerprints without the need for supervised learning pre-classification. Furthermore, the fingerprint can act as an unbiased benchmark for new methods. Finally, we show that this method, based on a simple set operation and hashing scheme, can perform better than both deep learning-based learned fingerprints and physicsbased descriptors in yield prediction tasks. We make the fingerprint creation algorithm available as a pypi package (drfp). The source code, data, and documentation are available on GitHub (https://github.com/reymond-group/drfp). 


\subsection{Reaction classification}

As a reaction classification task, we investigated the opensource USPTO 1k TPL data set, which we previously introduced. ${ }^{11}$ In USPTO $1 \mathrm{k}$ TPL, the reaction classes were generated by extracting the 1000 most common templates from the USPTO data set. $^{22}$ Atom-maps that are required to extract templates were predicted using RXNMapper. ${ }^{23}$ The task is to predict the corresponding template class given a chemical reaction.

The reaction classification was carried out using the $k$ nearest neighbor classifier based on faiss ${ }^{24}$ as defined by Schwaller et al. ${ }^{11}$ Initially, different versions of DRFP were evaluated on the USPTO $1 \mathrm{k}$ TPL set using a number of different configurations, namely for radius $r \in\{2,3,4\}$ and dimensionality $d \in\{16,32,64,128,256,512,1024,2048\}$. For all chosen radii, the accuracy increases strongly between $d=16$ to $d=128$, while only increasing slightly from $d=256$ to $d=2048$. The $r=$ 2 variant performs better than $r \in\{3,4\}$ for $d \in\{16,32\}$ (Fig. 2a). This is due to fewer collisions during mod hashing resulting from fewer extracted sub-structures. Starting with $d=256$, the $r$ $=3$ variant performs better than both the other variants.

Reducing the training set to 10 and $1 \%$ of its original size, aside from a general reduction in accuracy, also leads to a better relative performance of the $r=2$ variant across all dimensions $d$ (Fig. 2b and c). These results suggest that choosing the $r=2$ variant might be advantageous in low data settings, and there is no value in choosing $r=4$ over $r=2$ or $r=3$, independent from $d$ and the amount of available training data. However, as the $r=$ 3 variant performed best in the case of the complete training set for high $d$, the $r=3$ and $d=2048$ variant is chosen for all further benchmarks, including reaction yield predictions.

Table 1 shows the classification accuracy of DRFP on the USPTO $1 \mathrm{k}$ TPL data set compared to the structure-based fingerprint AP3 256 and the learned fingerprint rxnfp. ${ }^{11,17}$ Evaluating the $k$-nearest neighbour classification benchmark on the TPL data set, DRFP outperforms the structure-based fingerprint AP3 256 by a factor of 3.1 and reaches $93 \%$ of the performance of the learned fingerprint rxnfp. In addition, a variant of DRFP that

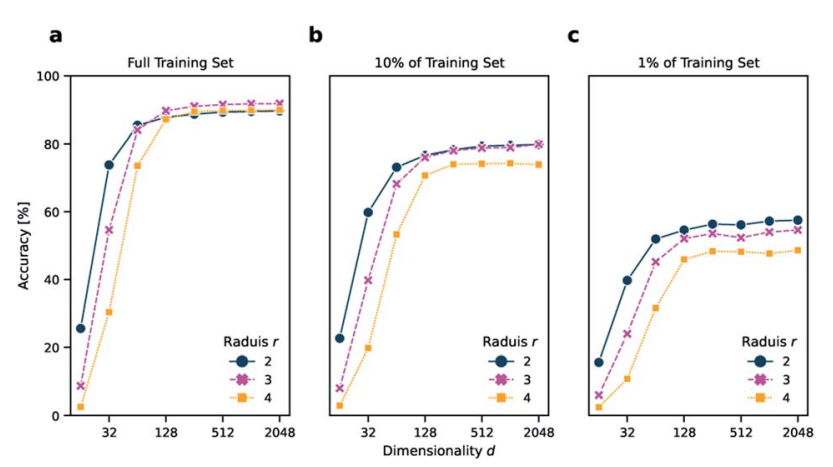

Fig. 2 Accuracy of the $k$-nearest neighbor classification on (a) the entire TPL data set, (b) $10 \%$ of the data set, and (c) $1 \%$ of the data set using DRFP fingerprints for dimensionality $d \in\{16,32,64,128,256$, $512,1024,2048\}$ and $r \in\{2,3,4\}$. The accuracy starts to plateau with $d=128$ independently from the amount of training data. However, a lower $r$ increases the accuracy in low data settings and when a low dimensionality $d$ is chosen due to increased generality and fewer collisions, respectively.
Table 1 Reaction classification accuracy on the USPTO 1k TPL data set

\begin{tabular}{lllll}
\hline USPTO 1k TPL & Classifier & Accuracy & CEN & MCC \\
\hline rxnfp & 5-NN & $\mathbf{0 . 9 8 9}$ & 0.006 & 0.989 \\
AP3 256 & 5-NN & 0.295 & 0.242 & 0.292 \\
DRFP (subtraction) & 5-NN & 0.851 & 0.850 & 0.074 \\
DRFP & 5-NN & 0.917 & 0.041 & 0.917 \\
AP3 256 & MLP & 0.809 & 0.101 & 0.808 \\
DRFP & MLP & 0.977 & 0.011 & 0.977
\end{tabular}

mimics the subtraction method of AP3 256 is evaluated, performing better than AP3 256 but not reaching the performance of the symmetric difference-based variant of DRFP. Replacing the $k$ nearest neighbour classifier with a simple multilayer perceptron (MLP) for DRFP and AP3 256, DRFP reaches 99\% of the performance of rxnfp, while AP3 256 only reaches $82 \%$. This result suggests that conceptual complexity, including learning, can be factored out of fingerprint creation, moving it instead to the classification task with a minor impact on classification performance. A non-learned fingerprint has the advantages of reducing bias and increasing the interpretability of results as each feature can be mapped to one or more molecular substructures.

Inspired by the rxnfp-based reaction atlas from our previous work, we created a similar TMAP ${ }^{25}$ for the Schneider $50 \mathrm{k}$ data set using DRFP. ${ }^{11,17}$ The Schneider data set contains 50000 reactions that are distributed evenly over 50 reaction classes, as annotated by the NameRxn tool. ${ }^{16}$ To analyse the performance of DRFP on the data set, we ran a classification task using the architecture and hyperparameters from the MLP used to classify the USPTO $1 \mathrm{k}$ TPL data set. After training on 10000 reactions and evaluating on the remaining 40000 , the model reached an average classification accuracy of $0.956(\mathrm{CEN}=0.053, \mathrm{MCC}=0.955)$. A confusion matrix across the 50 reaction classes in the data set shows that the high classification accuracy holds across the majority of the classes (Fig. 3a). Similar to the rxnfp-based classifier, our model reaches the lowest accuracy for the methylation reaction class, as methylation reactants often cause misclassifications. ${ }^{\mathbf{1 1}}$ An example standing out in Fig. 3a are methylations involving iodomethane as a reagent with nitrogen-containing products being classified as iodo $\mathrm{N}$-alkylations. Indeed, Schneider et al. also identified these classes as a source of misclassification and attributed them to the fact that some of the ground truth class assignments are ambiguous. ${ }^{17}$ The clustering of reactions by their super-classes in the TMAP (Fig. 3b) further shows that DRFP is well-suited for reaction classification tasks.

\subsection{Reaction yield prediction}

As a reaction regression task, we investigated yield prediction, where, given a chemical reaction, the percentage of the product that is formed compared to the theoretical maximum has to be predicted. One of the best studied yield data sets comes from a high-throughput experimentation study by Ahneman et al., ${ }^{\mathbf{6}}$ which contains the yields of 4608 palladium-catalysed Buchwald-Hartwig reactions with a fixed reactant and varying reagents. Numerous studies have previously used this data set model to evaluate the different machine learning models and 

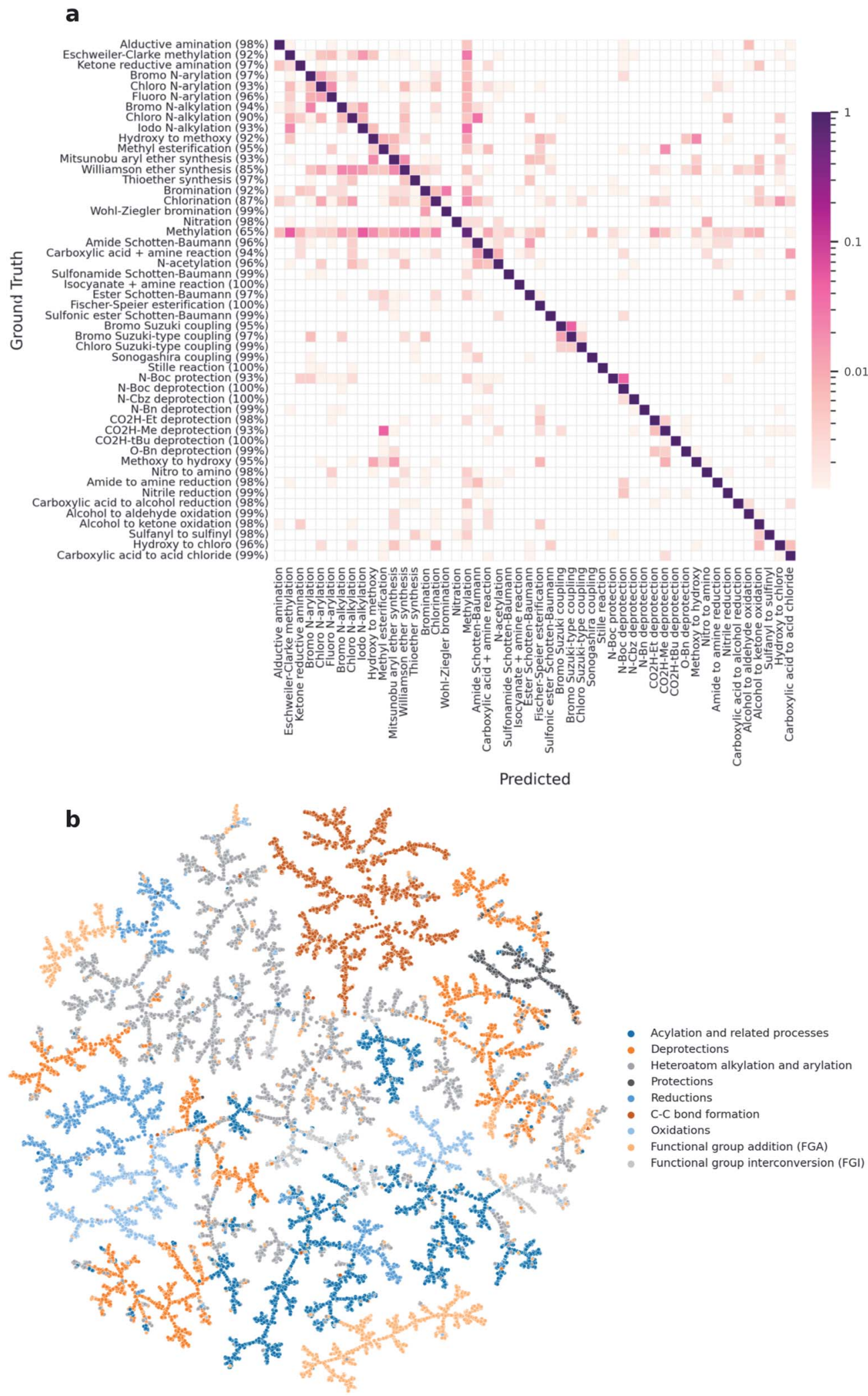

Fig. 3 (a) An analysis of the per-class classification accuracy of an MLP model trained on DRFP shows that the high accuracy holds across most reaction classes (correctly predicted percentage per class in parentheses). (b) A TMAP created from DRFP fingerprints shows reactions being clustered by their respective super-class. 
Table $2 R^{2}$ of yield prediction on Buchwald Hartwig reactions

\begin{tabular}{lllll}
\hline$R^{2}$ & DFT $^{6}$ & Yield-BERT $^{10}$ & ${\text { Yield-BERT (aug. })^{27}}$ \\
\hline Rand 70/30 & 0.92 & $0.95 \pm 0.005$ & $\mathbf{0 . 9 7} \pm \mathbf{0 . 0 0 3}$ & $0.95 \pm 0.005$ \\
Rand 50/50 & 0.9 & $0.92 \pm 0.01$ & $\mathbf{0 . 9 5} \pm \mathbf{0 . 0 1}$ & $\mathbf{0 . 9 2} \pm \mathbf{0 . 0 1}$ \\
Rand 30/70 & 0.85 & $0.88 \pm 0.01$ & $\mathbf{0 . 8 9} \pm \mathbf{0 . 0 1}$ & $0.93 \pm 0.01$ \\
Rand 20/80 & 0.81 & $0.86 \pm 0.01$ & $\mathbf{0 . 8 1} \pm \mathbf{0 . 0 2}$ & $0.89 \pm 0.01$ \\
Rand 10/90 & 0.77 & $0.79 \pm 0.02$ & $\mathbf{0 . 7 4} \pm \mathbf{0 . 0 3}$ & $0.61 \pm 0.04$ \\
Rand 5/95 & 0.68 & $0.61 \pm 0.04$ & $0.8 \pm 0.01$ & $0.73 \pm 0.02$ \\
Rand 2.5/97.5 & 0.59 & $0.45 \pm 0.05$ & $\mathbf{0 . 8 8} \pm \mathbf{0 . 0 2}$ & $\mathbf{0 . 6 2} \pm \mathbf{0 . 0 4}$ \\
Test 1 & 0.8 & $\mathbf{0 . 8 4} \pm \mathbf{0 . 0 1}$ & $0.56 \pm 0.08$ & $0.81 \pm 0.01$ \\
Test 2 & 0.77 & $0.84 \pm 0.03$ & $0.43 \pm 0.04$ & $0.83 \pm 0.003$ \\
Test 3 & 0.64 & $\mathbf{0 . 7 5} \pm \mathbf{0 . 0 4}$ & $0.58 \pm 0.33$ & $0.71 \pm 0.001$ \\
Test 4 & $\mathbf{0 . 5 4}$ & $0.49 \pm 0.05$ & $0.778 \pm 0.18$ &
\end{tabular}

Table $3 R^{2}$ of yield prediction on Suzuki Miyaura reactions and on the USPTO data set that has been divided into gram scale and sub-gram scale yield subsets

\begin{tabular}{lll}
\hline$R^{2}$ & Yield-BERT & DRFP (gradient boost) \\
\hline Suzuki Miyaura & $0.81( \pm 0.01)$ & $\mathbf{0 . 8 5}( \pm 0.01)$ \\
USPTO (gram scale) & 0.117 & $\mathbf{0 . 1 3}$ \\
USPTO (sub-gram scale) & 0.195 & $\mathbf{0 . 1 9 7}$
\end{tabular}

representations (one-hot, ${ }^{26}$ physical, $^{6}$ molecular ${ }^{\mathbf{1 9}}$ and learned ${ }^{\mathbf{1 0 , 1 1}}$ descriptors). The data set contains 10 random splits and 4 out-of-distribution test sets. In the out-of-distribution test sets, the split is made on the additives, which strongly influence the reactivity. Hence, the models have to extrapolate to unseen additives to perform well.

Comparing the yield prediction performance of DRFP to that of learned and physical descriptor-based fingerprints shows that this simple fingerprint is competitive, as it demonstrates consistent performance on all test sets. Averaging the 11 tests shown in Table 2, DRFP performs better than Yield-BERT, an augmented version of Yield-BERT, as well as a DFT-based method, in a yield prediction task on a data set of Buchwald Hartwig reactions. It also performs better than rxnfp in yield prediction of USPTO reaction data and a data set of Suzuki Miyaura reactions (Table 3).

In order to predict reaction yields using $D R F P$, gradient boosting with early stopping was chosen as a machine learning technique. $10 \%$ of each training split was set aside and used to evaluate for early stopping. Hyperparameter optimisation was performed on five random splits (70/30). The resulting performance $\left(R^{2}\right)$ is then compared to the density functional theory (DFT) based fingerprint with a random forest regressor by Ahneman et al., ${ }^{6}$ Yield-BERT, an extension of the learned rxnfp fingerprint with a regression layer, and an augmented variant of the latter (Table 2). The data set used is a collection of 3955 Pdcatalysed Buchwald-Hartwig $\mathrm{C}-\mathrm{N}$ cross-coupling reactions from a high throughput experiment by Ahneman et al. ${ }^{6}$ For this data set, 11 splits were defined; seven splits where the relative size of the training set was decreased from 70 to $2.5 \%$ and four out-ofsample splits based on isoxazole additives. DRFP performs better on the random splits than the DFT-based fingerprint with random forests and Yield-BERT but is outperformed by the augmented Yield-BERT by a narrow margin. In the out-ofsample splits, DRFP performs better than the augmented version of Yield-BERT and the DFT-based method, yet the nonaugmented Yield-BERT performs slightly better. When averaging over all 11 tests, DRFP performs best. Fig. 4 shows the regression plots for both the random split (a-g) and the out-ofsample (i-l) experiments. Under a low data regime, the xgboost model trained on DRFP tends to overestimate low-yield reactions and underestimate high-yield reactions (Fig. $4 \mathrm{a}-\mathrm{c}$ ), while the augmented Yield-BERT model generally predicts yields that are too low for low-yield reactions and too high for high-yield reactions. ${ }^{27} \mathrm{~A}$ similar tendency can be seen for the out-ofsample splits (Fig. 4a-c).

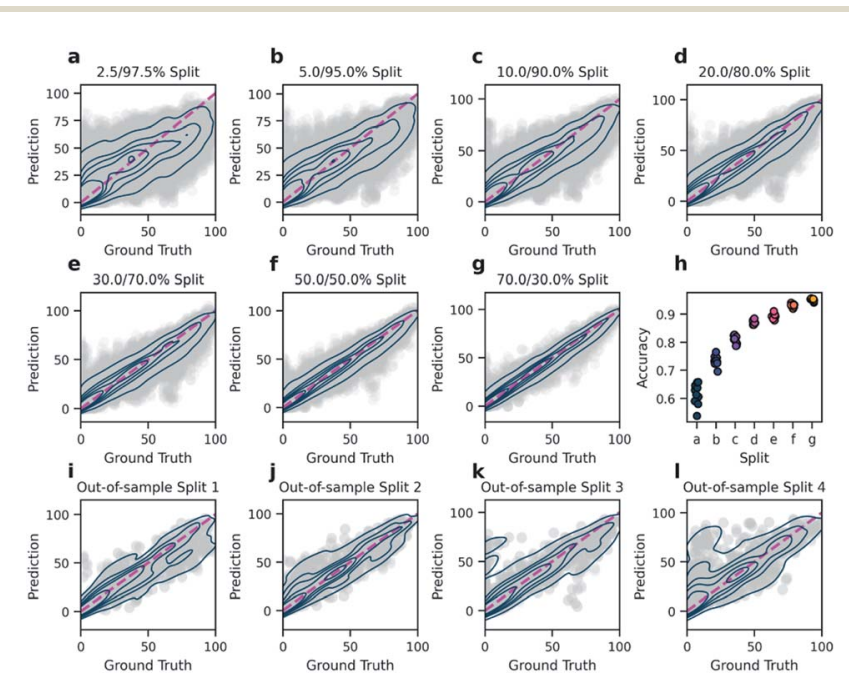

Fig. 4 Regression plots for both the random split $(a-g)$ and the outof-sample $(i-l)$ experiments. With few training data, the model generally predicts yields that are too high for low-yield reactions and yields that are too low for high-yield reactions $(a-c)$. Further increase in the size of the training data yields diminishing increases in accuracy while the variance of accuracy between test sets decreases $(d-h)$. In the out-of-sample-tests where the splits are defined by the isoxazole additives, performance varies heavily $(\mathrm{i}-\mathrm{l})$. 
The performance of $D R F P$ was further tested on a data set containing the yields of Suzuki-Miyaura reactions from a highthroughput experiment, and on reactions with associated yields from the USPTO reaction data set. The USPTO yield data set was split into a sub-gram and gram set to account for the different distributions of yields in the two subsets, as described by Schwaller et al. ${ }^{10}$ On both data sets, DRFP performed better than Yield-BERT (Table 3). Similar to the Buchwald-Hartwig reaction data, the difference between the two approaches is relatively small. In the case of the USPTO data set, both methods perform better on reactions with a sub-gram scale yield. A current limitation of $D R F P$ is that it fails to distinguish between a reaction and its reverse, e.g. $\mathrm{A}+\mathrm{B} \rightarrow \mathrm{C}+\mathrm{D}$ and $\mathrm{C}+\mathrm{D} \rightarrow \mathrm{A}+\mathrm{B}$. However, as the direction of the reaction is usually implied by the presence and absence of reactants, we consider this to be an edgecase that, if necessary, could be addressed in a specialised variant of the fingerprint.

Overall, DRFP reaches a compelling performance in yield prediction using a gradient boosting regressor that does not require hyperparameter tuning between different sets.

\section{Conclusion}

We have introduced a reaction fingerprint encoding scheme, $D R F P$, based on a simple 4-step process comprised of extracting circular $n$-grams, XORing, hashing, and folding. DRFP is capable of reaching state-of-the-art performance without extending the use of machine learning models from classification or regression tasks to the fingerprint creation task. Our results show that SMILES-encoded molecular graphs contain information that is sufficient for yield prediction by simple machine learning models, and that the explicit calculation of physics-based descriptors from the molecular graph as carried out by Ahnemann et al. is not necessary for this prediction task. While our method only slightly improves on the classification and prediction accuracies of other state-of-the-art methods, its value lies within its conceptual simplicity, low use of computational resources, and reproducibility. The fingerprint creation algorithm is available as a pypi package (drfp). Source code and documentation are available on GitHub (https:/github.com/ reymond-group/drfp).

\section{Methods}

\subsection{Computational resources}

We ran all of the training runs as well as the evaluations of the models on a DELL XPS Laptop with 16 GB of main memory, no dedicated GPU, and an 11th Gen Intel(R) Core(TM) i7-1165G7 @ $2.80 \mathrm{GHz}$ CPU.

\subsection{Molecular $n$-grams}

Molecular $n$-grams are generated from SMILES, text-based encodings of the molecular graph, using the RDKit library. Given a radius $r$, we iterate over the heavy atoms in an input molecule and extract substructures centred on each atom with radii 0 to $r$, where a radius of 0 is the single central atom. These extracted substructures are then encoded as SMILES. A visual representation of this process for one atom in a molecule and $r$ $=3$ is shown in Fig. 1c. We denote the SMILES encodings of extracted substructures molecular $n$-grams in reference to $n$ grams found in bioinformatics. ${ }^{28}$ In addition, rings from the SSSR (smallest set of smallest rings) are extracted as $n$-grams as well. Compared to the atom pair-based approach by Schneider et $a l .,{ }^{17}$ the $n$-grams-based fingerprint also captures stereochemistry, which can be defined using the SMILES notation. The pseudo-code for this process is shown in Box 1.

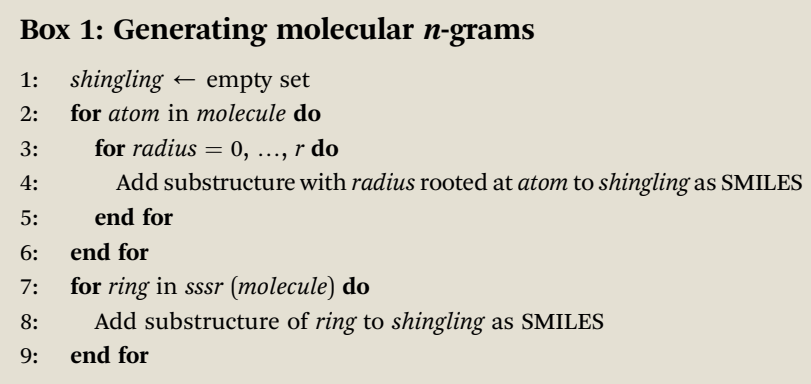

\subsection{Gradient boosting}

For regression by gradient boosting, we used the Python library xgboost. Hyperparameter tuning was carried out on the rand 70/ 30 set of the Buchwald-Hartwig reaction data set. We applied the same hyperparameter values (n_estimators $=999999$, learning_rate $=0.01$, max_depth $=15$, min_child_weight $=8$, colsample_bytree $=0.2125$, subsample $=1$ ) in all uses of xgboost. For each test, $10 \%$ of the training data were randomly selected as the validation set an removed from the training set. The validation data sets were used as evaluation sets for early stopping (20 rounds for all data sets with the exception of the USPTO, data for which 10 rounds were used to speed up the calculation).

\section{4 $k$-Nearest neighbours classifier}

The $k$-nearest neighbour classifier was implemented according to Schwaller et al. ${ }^{11}$ using faiss with $k=5$.

\subsection{Multilayer perceptron classifier}

In addition to $D R F P+5$-NN classifier, $D R F P+$ multilayer perceptron (MLP) classifier was applied to the USPTO $1 \mathrm{k}$ TPL data set. The MLP was implemented using Tensorflow 2.4.1 and consists of an input layer the size of the input vector $(2,048)$, a dense hidden layer of size 1664 and a tanh activation function, and a dense output layer with a softmax activation function. The loss function was sparse categorical cross-entropy. Adam was used as an optimiser. The model was trained over 10 epochs with a batch size of 64 on a CPU.

For the evaluation of AP3 256, the number of units in the hidden layer was changed to 1024, and the model was trained for 100 epochs. 


\subsection{Visualisations}

All plots were created using the Python library matplotlib, ${ }^{29}$ the confusion matrix for the Schneider $50 \mathrm{k}$ classification task (Fig. 3a) was generated using the Python package pycm, ${ }^{30}$ and the TMAP coordinates for the Schneider 50k classification task (Fig. 3b) was created using the TMAP Python library. ${ }^{25}$

\section{Data availability}

The source code, data and processing scripts for this paper, including the scripts to generate the fingerptins and the models are available at https://github.com/reymond-group/drfp. An release associated with the manuscript has been uploaded to Zenodo under the record https://zenodo.org/record/ 5268144\#.YSeDXFuxWAk with DOI: 10.5281/zenodo.5268144.

\section{Author contributions}

Daniel Probst: writing - original draft, visualization, software, formal analysis, investigation, methodology, validation Philippe Schwaller: writing - original draft, data curation, validation Jean-Louis Reymond: writing - review and editing, supervision, funding acquisition, project administration, resources.

\section{Conflicts of interest}

There are no conflicts of interest to declare.

\section{Acknowledgements}

This work was supported financially by the Swiss National Science Foundation, NCCR TransCure.

\section{Notes and references}

1 P. Raccuglia, K. C. Elbert, P. D. F. Adler, C. Falk, M. B. Wenny, A. Mollo, M. Zeller, S. A. Friedler, J. Schrier and A. J. Norquist, Nature, 2016, 533, 73-76.

2 G. Skoraczyński, P. Dittwald, B. Miasojedow, S. Szymkuć, E. P. Gajewska, B. A. Grzybowski and A. Gambin, Sci. Rep., 2017, 7, 3582.

3 C. W. Coley, L. Rogers, W. H. Green and K. F. Jensen, ACS Cent. Sci., 2017, 3, 1237-1245.

4 M. H. S. Segler, M. Preuss and M. P. Waller, Nature, 2018, 555, 604-610.

5 P. Schwaller, T. Gaudin, D. Lányi, C. Bekas and T. Laino, Chem. Sci., 2018, 9, 6091-6098.

6 D. T. Ahneman, J. G. Estrada, S. Lin, S. D. Dreher and A. G. Doyle, Science, 2018, 360, 186-190.

7 A. C. Vaucher, F. Zipoli, J. Geluykens, V. H. Nair, P. Schwaller and T. Laino, Nat. Commun., 2020, 11, 3601.
8 N. S. Eyke, W. H. Green and K. F. Jensen, React. Chem. Eng., 2020, 5, 1963-1972.

9 Z. Fu, X. Li, Z. Wang, Z. Li, X. Liu, X. Wu, J. Zhao, X. Ding, X. Wan, F. Zhong, D. Wang, X. Luo, K. Chen, H. Liu, J. Wang, H. Jiang and M. Zheng, Org. Chem. Front., 2020, 7, 2269-2277.

10 P. Schwaller, A. C. Vaucher, T. Laino and J.-L. Reymond, Mach. Learn.: Sci. Technol., 2021, 2, 015016.

11 P. Schwaller, D. Probst, A. C. Vaucher, V. H. Nair, D. Kreutter, T. Laino and J.-L. Reymond, Nat. Mach. Intell., 2021, 3, 144152.

12 J. S. Carey, D. Laffan, C. Thomson and M. T. Williams, Org. Biomol. Chem., 2006, 4(12), 2337-2347.

13 S. D. Roughley and A. M. Jordan, J. Med. Chem., 2011, 54, 3451-3479.

14 RSC Ontologies, rxno.

15 H. Kraut, J. Eiblmaier, G. Grethe, P. Low, H. Matuszczyk and H. Saller, J. Chem. Inf. Model., 2013, 53, 2884-2895.

16 https:/www.nextmovesoftware.com/namerxn.html.

17 N. Schneider, D. M. Lowe, R. A. Sayle and G. A. Landrum, J. Chem. Inf. Model., 2015, 55, 39-53.

18 G. M. Ghiandoni, M. J. Bodkin, B. Chen, D. Hristozov, J. E. A. Wallace, J. Webster and V. J. Gillet, J. Chem. Inf. Model., 2019, 59, 4167-4187.

19 F. Sandfort, F. Strieth-Kalthoff, M. Kühnemund, C. Beecks and F. Glorius, Chem, 2020, 6, 1379-1390.

20 D. Rogers and M. Hahn, J. Chem. Inf. Model., 2010, 50, 742754.

21 D. Probst and J.-L. Reymond, J. Cheminf., 2018, 10, 66.

22 D. Lowe, Chemical reactions from US patents (1976Sep2016), figshare, Dataset, 2017, DOI: 10.6084/ m9.figshare.5104873.v1.

23 P. Schwaller, B. Hoover, J.-L. Reymond, H. Strobelt and T. Laino, Sci. Adv., 2021, 7, eabe4166.

24 J. Johnson, M. Douze and H. Jégou, 2017, arXiv preprint arXiv:1702.08734.

25 D. Probst and J.-L. Reymond, J. Cheminf., 2020, 12, 12.

26 K. V. Chuang and M. J. Keiser, Science, 2018, 362(6416), eaat8603.

27 P. Schwaller, A. C. Vaucher, T. Laino and J.-L. Reymond, 2020, ChemRxiv, preprint, DOI: 10.26434/ chemrxiv.13286741.v1.

28 A. Tomović, P. Janičić and V. Kešelj, Comput. Methods Progr. Biomed., 2006, 81, 137-153.

29 J. D. Hunter, Comput. Sci. Eng., 2007, 9, 90-95.

30 S. Haghighi, M. Jasemi, S. Hessabi and A. Zolanvari, PyCM: Multiclass confusion matrix library in Python, Open J., 2018, 3, 729-729. 